\title{
QA/QC OF GAMMA-GAMMA GEOPHYSICAL WELL LOGGING DATA APPLIED TO THE CHARACTERIZATION OF HIGH DENSITY ROCK MASSES
}

\author{
Wanderson Roberto Pereira and Dionisio Uendro Carlos
}

\begin{abstract}
This paper proposes a set of procedures for quality control of gamma-gamma geophysical well logging data applied to the study of high density rock masses, which: (1) linearity verification between different spaced gamma-gamma sensors; (2) verification of the recorded densities range; (3) comparison between the internal diameters of the borehole walls and the nominal drilling diameter; and (4) verification of the proportionality between the densities measured by the sensors of short and long spacing by means of residual analysis. The quality control allows to mitigate any omissions of data interpretation (human factor), and to minimize the computational time, as well as facilitate any changes to the quality control criteria.
\end{abstract}

Keywords: geophysical well logging, gamma-gamma density, quality control.

RESUMO. 0 presente trabalho propõe um conjunto de procedimentos para controle de qualidade de dados de perfilagem geofísica gama-gama aplicados ao estudo de maciços rochosos de alta densidade, sendo: (1) verificação da linearidade entre sensores de perfilagem gama-gama; (2) verificação do intervalo de valores de densidades registradas; (3) comparação entre os diâmetros internos nas paredes dos furos de sondagem e o diâmetro nominal; e (4) verificação da proporcionalidade entre as densidades medidas pelos sensores de espaçamento curto e longo por meio da análise de resíduos. 0 controle de qualidade permite mitigar eventuais omissões decorrentes de fatores humanos na interpretação dos dados, bem como viabiliza eventuais mudanças nos critérios de verificação da qualidade dos dados.

Palavras-chave: perfilagem geofísica, densidade gama-gama, controle de qualidade.

Vale S.A., Exploração Mineral e Geologia, Centro de Tecnologia de Ferrosos - CTF, Fazenda Rio de Peixe s/n, Zona Rural, 34000-000 Nova Lima, MG, Brazil. Phone: +55(31) 3215-4301/3215-4201 - E-mails: wanderson.pereira@vale.com; dionisio.carlos@vale.com 


\section{INTRODUCTION}

Iron formations commonly present high values of density contrasts with respect to the hosting environment. In this context, rock density is a key parameter in the estimation of resources and reserves that contribute to the geological modeling activities for the purposes of iron ore exploration (Amigun \& Ako, 2009).

With respect to conventional techniques for determining the density of rocks (Balco \& Stone, 2003), gamma-gamma geophysical well logging is noteworthy (Telford et al., 1990) for presenting some advantages with respect to the method of direct measurement of density in drilling cores or samples.

In the gamma-gamma geophysical well logging the density measurements are performed inside the borehole (in-situ) and the record of these density measurements is acquired in a centimeters acquisition rate, preventing the ore volume from overestimating, unlike samples, where the interstitial spaces present in the rock mass and the scale factor are not considered.

The gamma-gamma geophysical well logging applied to the exploration of iron ore presents some peculiarities as ambiguity of densities reading between different rock types, both iron formation and the surrounding rocks and igneous intrusions, and the direct relation between the measurement quality conditioned by the characteristics of the rock mass, whether loose or compact, of more or less homogeneous composition, and with the presence of structural features throughout the borehole, or mechanically induced stress by the geological drilling activity.

For the gamma-gamma well logging data to be used systematically, it is necessary to distinguish sections with good signal recovery, from those whose measure is affected by operational and geological conditions. In the specific case of iron ore exploration, gamma-gamma data quality check is limited to the verification of caliper, for induced density variations.

In this paper, an innovative quality assessment and quality control methodology is proposed, along with a structured data quality analysis procedure.

\section{METHODOLOGY}

\section{Determination of Densities by the Geophysical Method of Gamma-gamma Well Logging}

The gamma-gamma well logging is based on the interaction of gamma radiation with matter, specifically in the manner how a particular material is crossed by a gamma ray beam (Ellis \& Singer, 2008) mainly in the energy range that promotes Compton scattering (Kaplan, 1964), approximately $600 \mathrm{keV}$.

For this purpose, a radioactive source of known activity,
${ }^{137} \mathrm{Cs}$, is used, emitting gamma radiation, and a set of sensors positioned under a controlled geometry (Fig. 1) which accumulate gamma particles that go through the subject material (Ellis \& Singer, 2008).

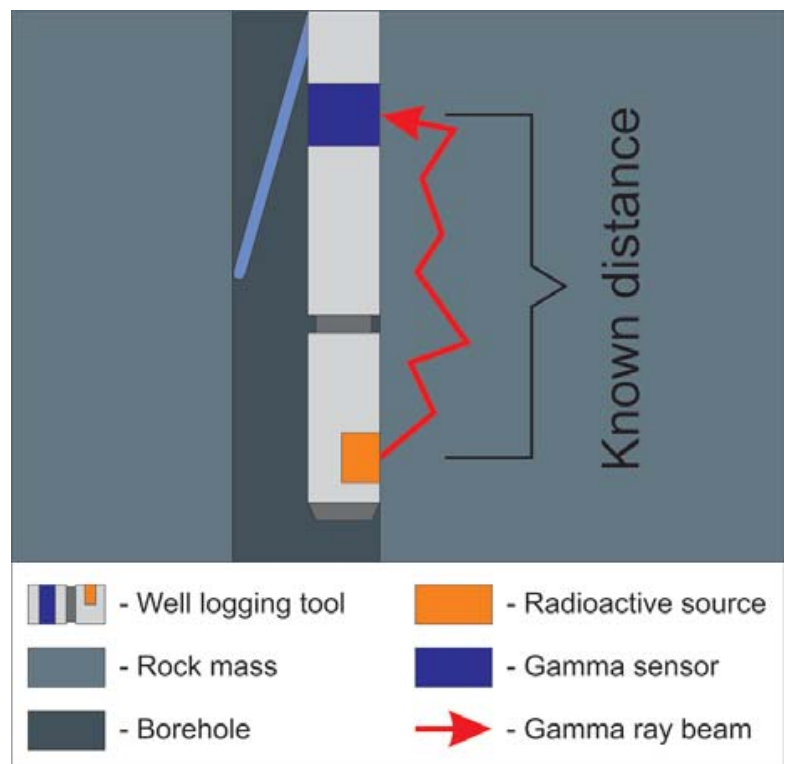

Figure 1 - Source-sensor array in gamma-gamma well logging.

Due to the interaction between gamma radiation and the geological material, the counting observed by the sensor is attenuated with respect to the counting observed directly from the radioactive source, and associate with the density of the geological material according to

$$
N=N_{0} e^{-\mu \rho x}
$$

where $N$ is the counting on the sensor, $N_{0}$ is the direct counting from the source, $\mu$ is the mass absorption coefficient, $\rho$ the material density, and $x$ the source-sensor distance (Ellis \& Singer, 2008).

By knowing the parameters involved in (1), it is possible to relate the density values to the values of gamma particles counting.

\section{Well Logging Operations}

The operations of gamma-gamma well logging consist in introducing a well logging probe in a borehole, which is equipped with a radioactive source and two receiver sensors for reading the counting per second (cps) of incident gamma particles with different spacing from the source, for determination of rock contacts influenced by proximity to each sensor as shown in Figure 2a.

Due to the nature of the operation, and the associated environmental constraints, such as instability of the bore walls, 


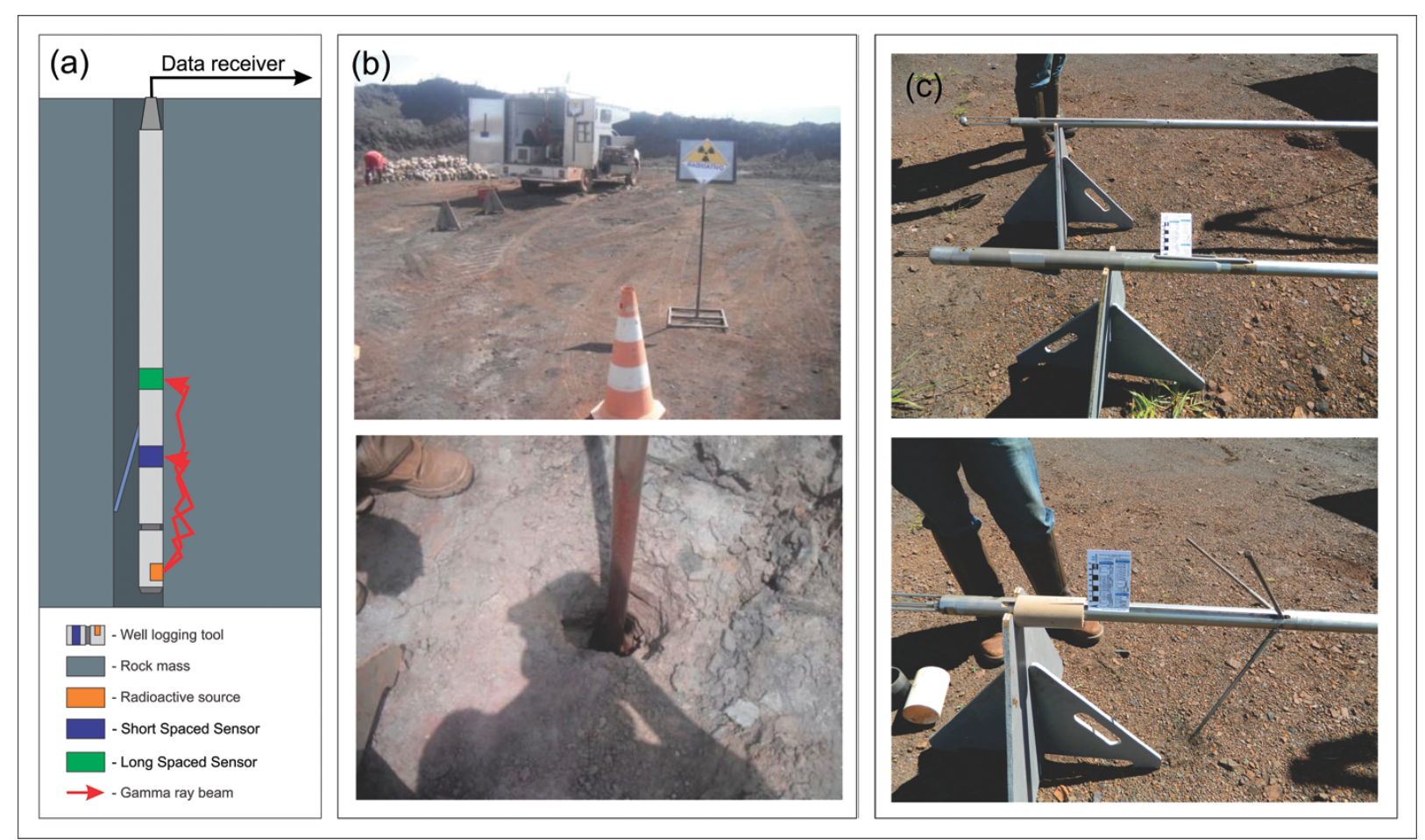

Figure 2 - a) Schematic layout of a gamma-gamma well logging probe operating in the borehole. b) Gamma-gamma well logging operation in a near-mine area. c) Examples of well logging tools.

some particular care must be taken in geophysical well logging intended for iron ore exploration, especially when this involves the use of a gamma-gamma tool.

Initially, it should be considered that in the case of open hole (boreholes without casing) there may be collapsing of the walls, particularly when it crosses ranges of friable rock.

To avoid accidents with radioactive source, before run the gamma-gamma tool, the borehole conditions are verified with a caliper tool that measures the variation in internal diameter along the hole, equipped with a natural gamma ray sensor.

In case of rocks with predominance of friable material, or with a history of collapse, it is advisable to run an inert tool, or dummy, without any radioactive source or measurement sensor. Such procedure avoids loss of well logging tool with radioactive source in the event of an accident, and the cost associated with the replacement of gamma-gamma well logging and radioactive source tool.

\section{Data Analysis}

The radiation intensity measured for a gamma ray source varies with the distance from the source (Morgan \& Turner, 1973), so, the first control in gamma-gamma density measurement is the distance between the source and the target, that is, the borehole wall.

The second control in gamma-gamma well logging data acquisition is the sensor position with respect to the radioactive source, and this to a conveniently located reference on top of the hole. This information is used to establish a reference for controlling the depth of each measure over the hole.

The gamma-gamma well logging information at a point over the hole, as well as the associated data should be arranged so that they have their relative positions well-defined, reason why the natural gamma tool must be attached to all other tools used, this way it uses the non-variable character of the natural radioactivity counting differences for the different geological materials, to calibrate the position of the various measurements made by different well logging tools.

Given a set of natural gamma data for two different tools in the same borehole, the depths adjustment may be made based on the overlap of the profiles (logs) around the outstanding features such as local peak counting as shown in Figure 3.

Another parameter that should be noted is the presence of water in the hole. Interstitial water will cause interference in the density values. To detect the presence of water, it is used a 


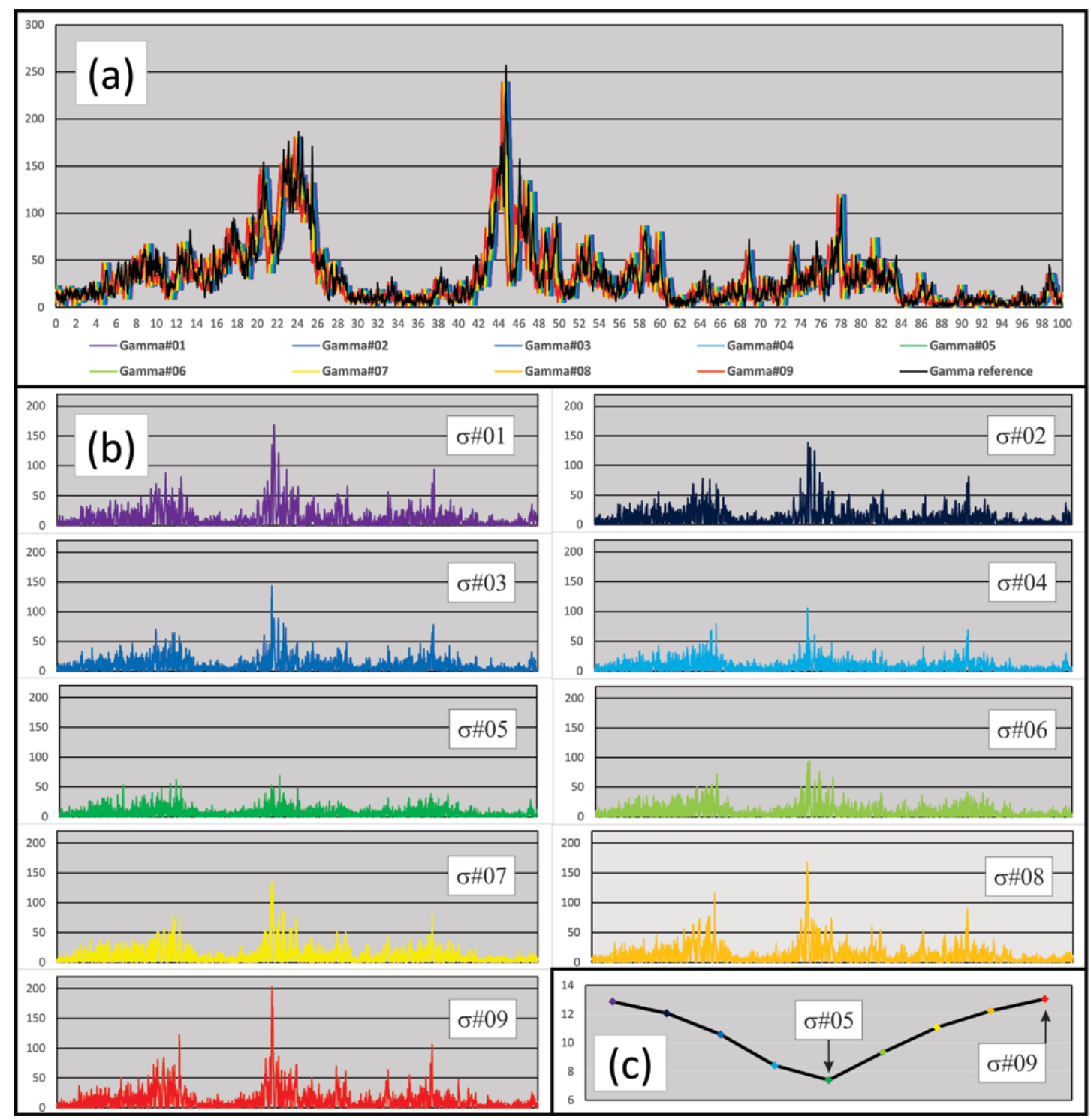

Figure 3 - Natural gamma count overlapping for two different tools in the same borehole. (a) Natural gamma dataset with nine different adjustments with relation to a common reference (b) Residuals showing the positions of lower adherence between two different tools ( $\sigma \# 01 ; \sigma \# 09)$, the positions with medium adherence ( $\sigma \# 02 ; \sigma \# 03 ; \sigma \# 04 ; \sigma \# 06 ; \sigma \# 07 ; \sigma \# 08)$ and the position of highest adherence $(\sigma \# 05)$. (c) Residual average per adjustment.

temperature sensor to indicate the water level in the hole, and, in the processing phase, a correction factor to compensate for the effect of the presence of water is applied.

For the analysis of gamma-gamma well logging data, there is a set of information contained in the profiles, these being the natural radioactivity of the rocks obtained from the natural gamma tool, the density of the rock obtained from gamma-gamma well logging, the temperature obtained through temperature sensor and variations in the hole walls diameter measured by the caliper tool.
Figure 4 shows an example of gamma-gamma log data, with the natural gamma data represented as a green filling log on the left, density data represented in the center in solid orange line, the temperature data represented to the right of the density profile, in cyan, and the caliper data represented to the right of the log in continuous blue line superimposed on the nominal diameter of the probing tool represented by the red solid line.

The logs must be presented with the appropriate depth adjustments based on natural gamma readings.

Based on a log containing the same information as the log 


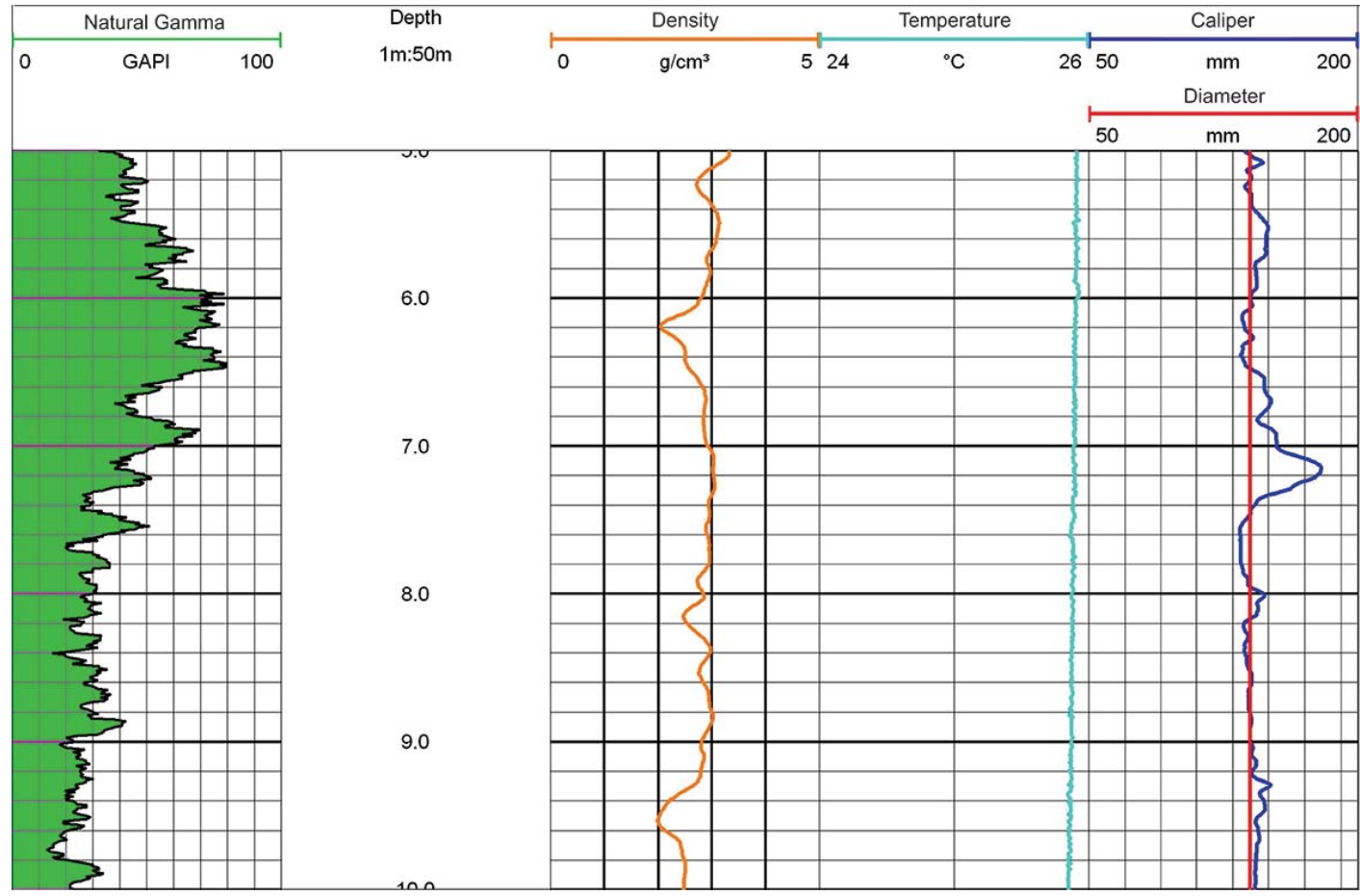

Figure 4 - Gamma-gamma well logging log with the essential dataset.

shown in Figure 4, and the well logging raw data, it shall be made the quality control of the gamma-gamma well logging data.

\section{Quality Control}

Quality control starts by checking the linearity in density readings obtained by the different sensors of the gamma-gamma tool, usually two, one long spaced (DLS) and other short spaced (DSS) (Fig. 2).

For a range of continuous and homogeneous lithology, with no significant structural or compositional variations such as discontinuities in rock mass and rock faulting, it is expected that both sensors read the same density, which does not occur in rock contacts where a layer influences one of the sensors more than the other, due to the proximity, as in transitions from lower to greater density (Fig. 5a), but it is found in homogeneous ranges (Fig. 5b) and presents the inverse expression from higher to lower density (Fig. 5c).

One may consider for a sharp contact between lithology that the transition between the densities values corresponding to each lithology, observed in the short spaced sensor is more significant than that found in the long spaced sensor, that is for the short sensor the transition is sharper, while for the long sensor the transition is smooth.

Such behavior can be verified experimentally both in the transition to a denser lithology as to a less dense lithology through the density readings around a point of convergence, that is, a thin layer of discordant density with respect to the adjacent densities for which both the sensor readings converge, it is possible to observe, for each sensor, a transition of input and output, as in the example of Figure 6.

In Figure 6, we observe, in addition to the decrease of the density values in the range of convergence, an increase in the natural gamma values, near 8 API between $6.0 \mathrm{~m}$ and $6.5 \mathrm{~m}$, coinciding with the convergence of input and output of the long spaced (DLS) and short spaced sensor (DSS) at the point indicated by the gray arrow.

In lithological contact zones, that is, ranges in the hole where contact occurs or transition between lithology, the tool can be positioned such that a sensor is in contact with a lithology and other sensor in contact with another lithology. In this case, a sensor records the density of a lithology and the other density composition thickness of both lithologies, and abnormal ranges of spacing less than the spacing between sensors are recorded only by one of the sensors, as shown in Figure 7.

Considering the geometry of the gamma-gamma well logging tool of Figure 2a, taking measurements during the ascent of the tool, the long spacing sensor is the first to record the density of an overlying lithology. 


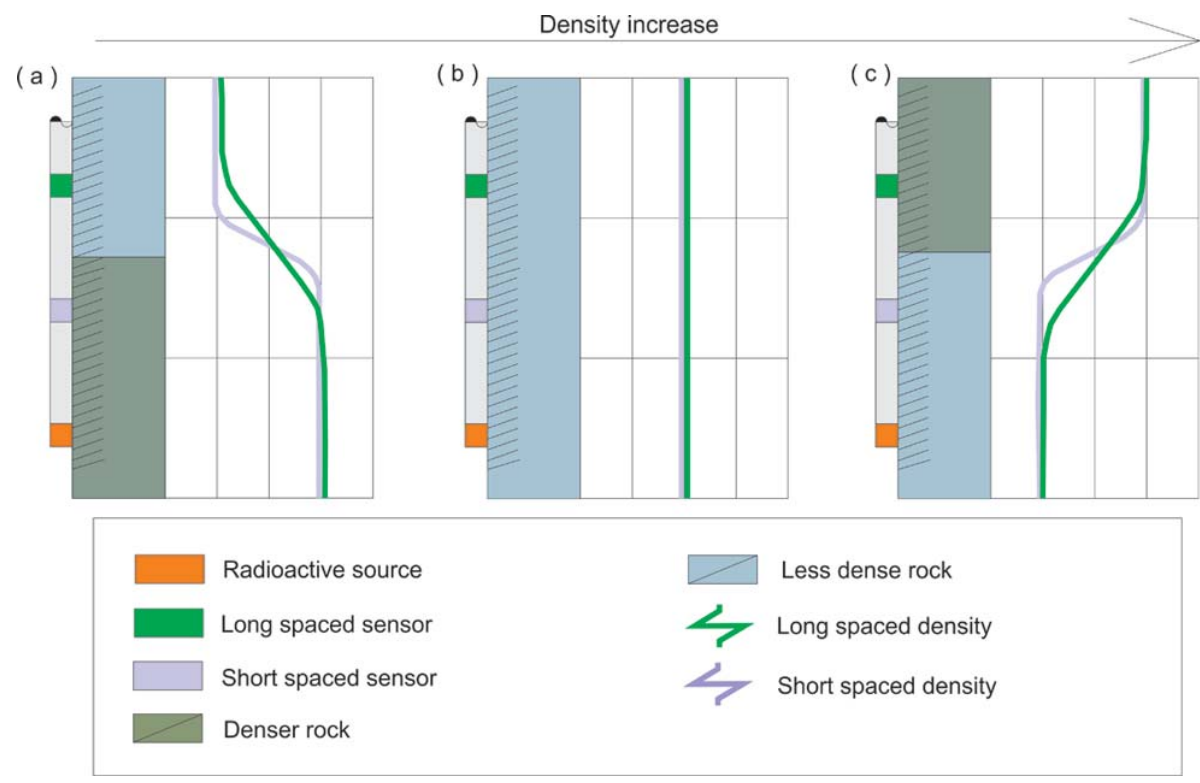

Figure 5 - Expected behavior for the density readings of the sensors inside and outside areas of geological contact. (a) Descending transition from less dense medium to denser medium. (b) Expected behavior for density in homogeneous medium. (c) Descending transition from denser medium to less dense medium.

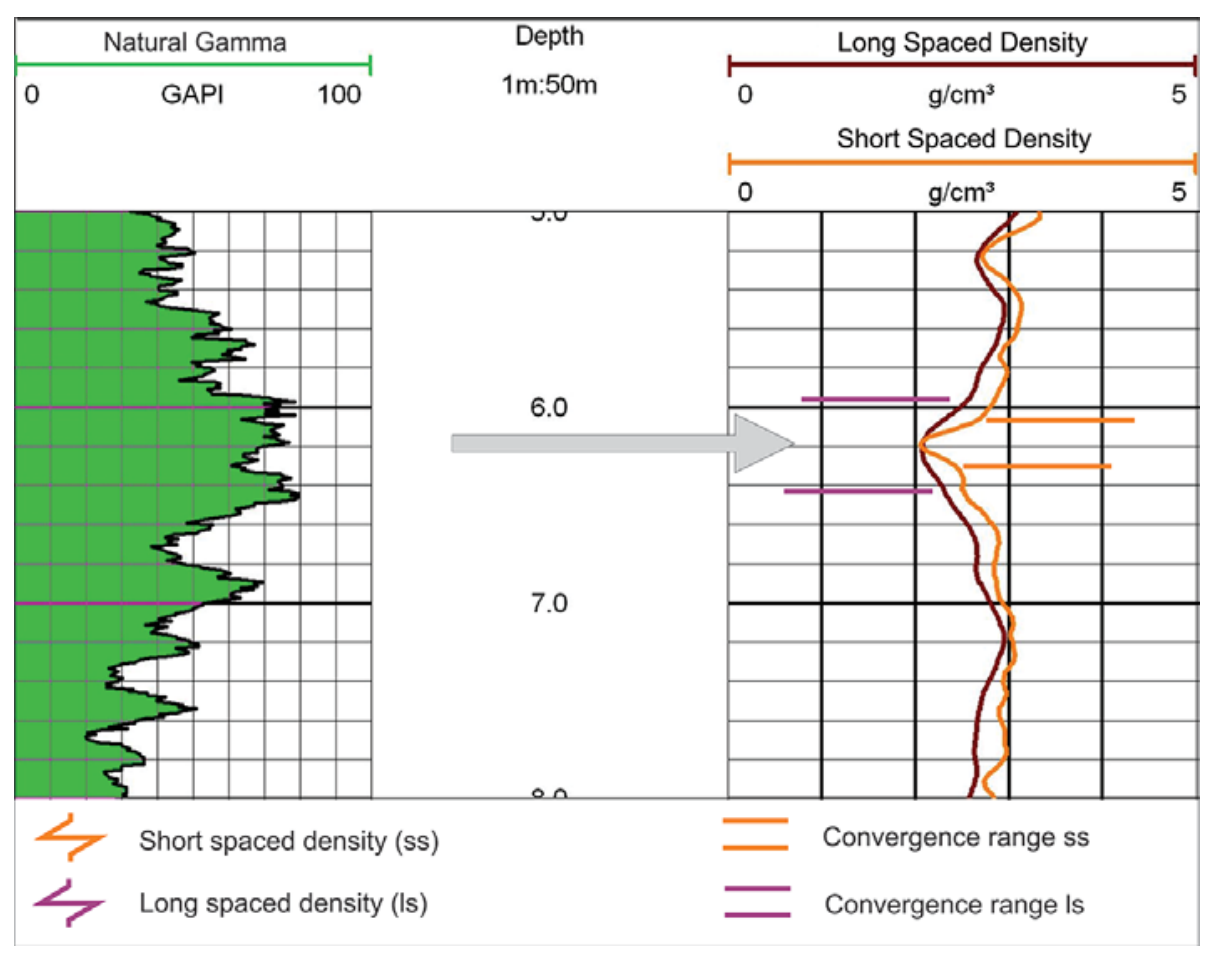

Figure 6 - Anomalous feature should range for which the convergence of input and output of the long spaced (DLS) and short spaced (DSS) sensors are different. The gray arrow indicates the zone of convergence where both densities converge to a value near $2.0 \mathrm{~g} / \mathrm{cm}^{3}$.

It is expected that there is consistency between the density values obtained by the two sensors, but thickness variations of the magnitude of the spacing between the sensors.
Figure 7 shows that between $8.0 \mathrm{~m}$ and $8.5 \mathrm{~m}$, the long spacing sensor (DLS) did not record the features indicated by the gray arrows, as it is recorded by the short spacing sensor (DSS), 


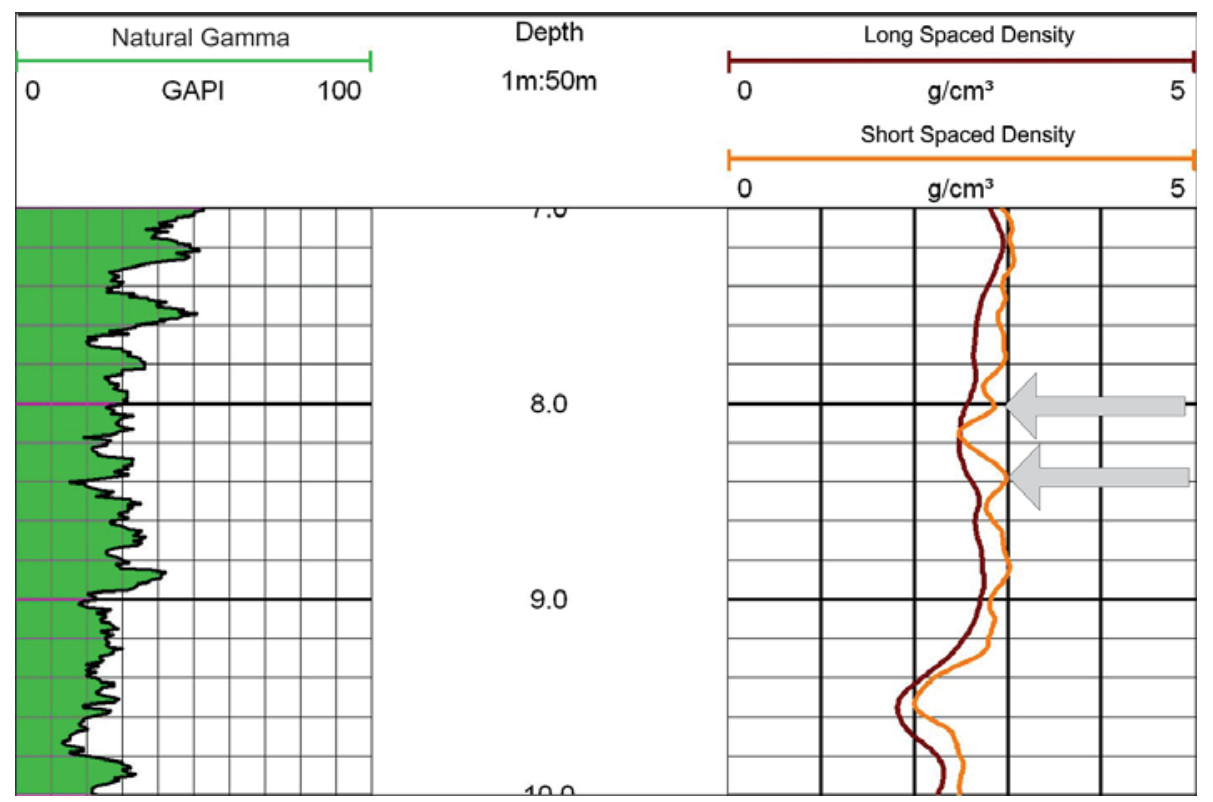

Figure 7 - Reading difference between the sensors readings to an anomalous occurrence of the magnitude of the spacing between sensors. The gray arrows indicate features read only by the short spacing sensor.

however, for the range between $7.0 \mathrm{~m}$ and $10 \mathrm{~m}$, the curves converge.

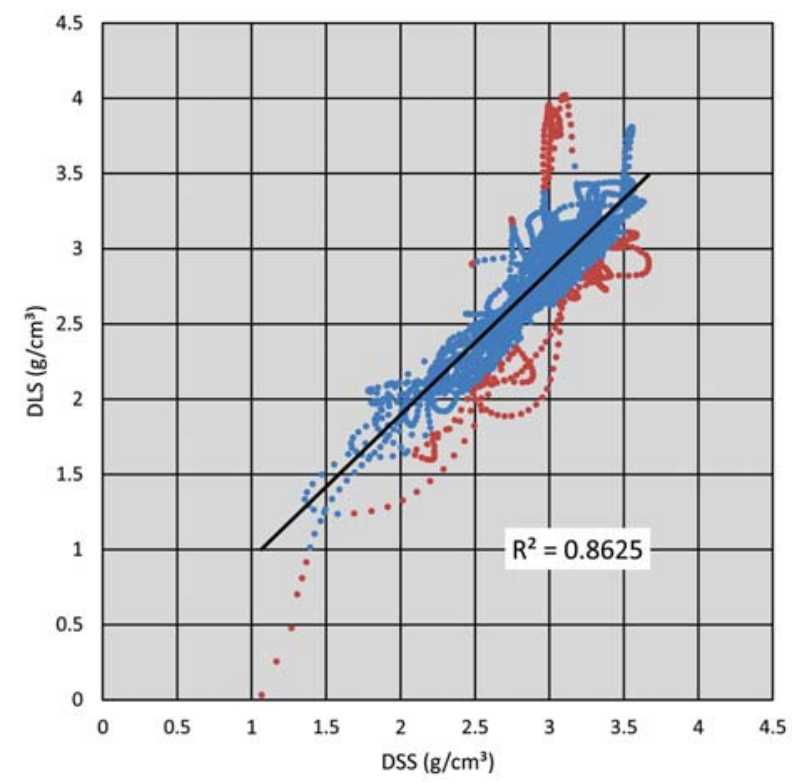

Figure 8 - The linearity between measures of short and long spaced sensors for a well logging dataset. DSS = densities measured by the short spaced sensor; $\mathrm{DLS}=$ densities measured by the long spaced sensor, and $R^{2}=$ correlation coefficient.

Thus, it can be set as the first check of gamma-gamma well logging data quality, a study of the linearity between the measurements of different sensors, for which is expected a linear behavior as illustrated in the graph of Figure 8, expected for the entire dataset, thus, the density measured by the long spacing sensor (DLS) presents a linear correlation with the short spacing (DSS).

The example of the Figure 8 shows the behavior of the measurements obtained by differently spaced gamma sensors of a same probe, showing a linear relation between them, with $R^{2}=$ 0.8 , for a $y=a x+b$ relation, where $a \cong 1$ and $b \cong 0$ equation type.

However, the linearity can be checked even if there is no geological consistency in the measurements, that is, both sensors, short and long spaced, may present measurements that, although correlated, diverge from the values expected to the geological context in which they are.

This may be due to problems in the tool communication, such as electronic data registry problems, as well as operational factors such as inadequate control of ascent rate. Either way, the range of density values obtained by the sensors must be checked throughout the length of the hole, which may require time proportional to the depth of the boreholes, considering an ascent rate of one meter per minute.

One way to optimize this check is to establish maximum and minimum densities, using a computational routine that reads data from a hole and compares densities of this record with the density limits established as expected for that geological context, or automatically delete data that is not between the limit values 


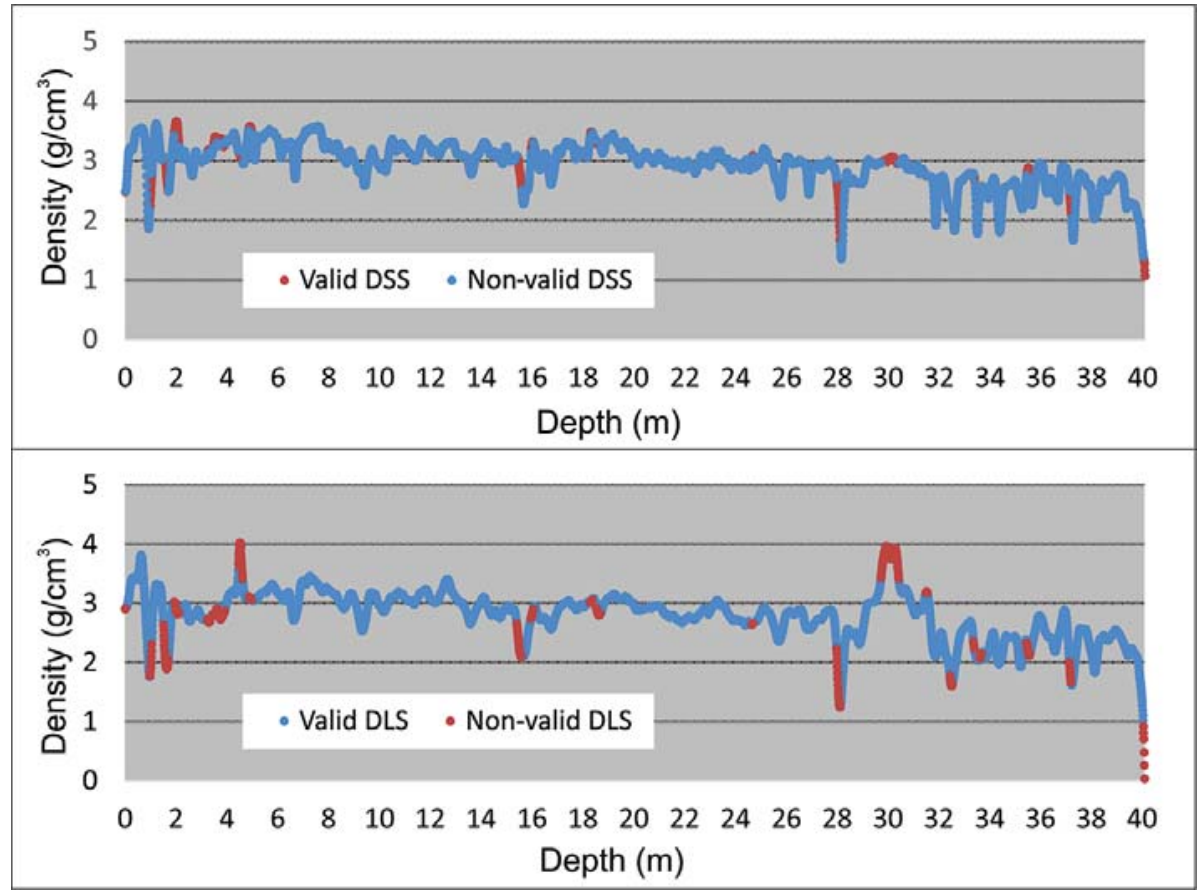

Figure 9 - Check of the gamma-gamma density range in the dataset of a hole deeper than 90 meters, recording over 9000 measures of density varying from $1.5 \mathrm{~g} / \mathrm{cm}^{3}$ and $3.6 \mathrm{~g} / \mathrm{cm}^{3}$.

established. The verification can be confirmed by viewing the set of density data as shown in Figure 9.

The densities range check of a hole is made at ranges, and constitutes the second proposed check for quality control of data from gamma-gamma well logging, with the exception that the analysis and eventual exclusion of range data, not being an entire hole discarded because of invalid ranges, as long as there is consistency in the valid ranges on a geological point of view.

Even with these two overlapping controls, whether they evaluate the linearity and range of densities, there may be ranges that, while complying with the requirements of the first and second check, still may not be valid for the interpretation of densities for presenting variations caused by mechanical factors related to the conditions of the borehole, such as collapses.

To attest the validity of density ranges, in this case, there is a variation in the borehole diameter measured by caliper tool, to verify compliance with the requirements of distance between the source and the target, for which there must be contact between the rock and sensor, and thus identify ranges whose borehole conditions may influence the density values.

Density values with intermittent or continuous variation may be related to both geological factors as composition and texture of the rock, natural mechanical factors such as faults and frac- tures, or induced mechanical factors such as fracturing caused by drilling activity, and these can be distinguished by comparing the density variation to the caliper curve.

The procedure consists in observing if the variation in the density profile has correspondence with any variation in the borehole wall, whereas in the case that such correspondence occurs, the variation of density may be attributed to the conditions verified on the borehole wall as shown in Figure 10.

In Figure 10, variations of caliper and respective density induced variations, indicated by the gray arrow between $62 \mathrm{~m}$ and $65 \mathrm{~m}$, have no correspondence with the natural gamma profile that shows no variation in the range, keeping below $10 \mathrm{API}$, which indicates no lithologic change associated.

Another possible correlation between these two parameters, roughness or variation in the diameter of the borehole wall and density may be verified in the case where the rock allows a degree of blistering, presenting decrease in the bore diameter relative to the drilling tool diameter in passages of loose rock, as exemplified in Figure 11:

The correspondence between the variations of caliper and density of Figure 11 may be associated with the rock feature to expand presenting, however, lower density. It is expected, in this case, that the compact rock presents a higher density and that 


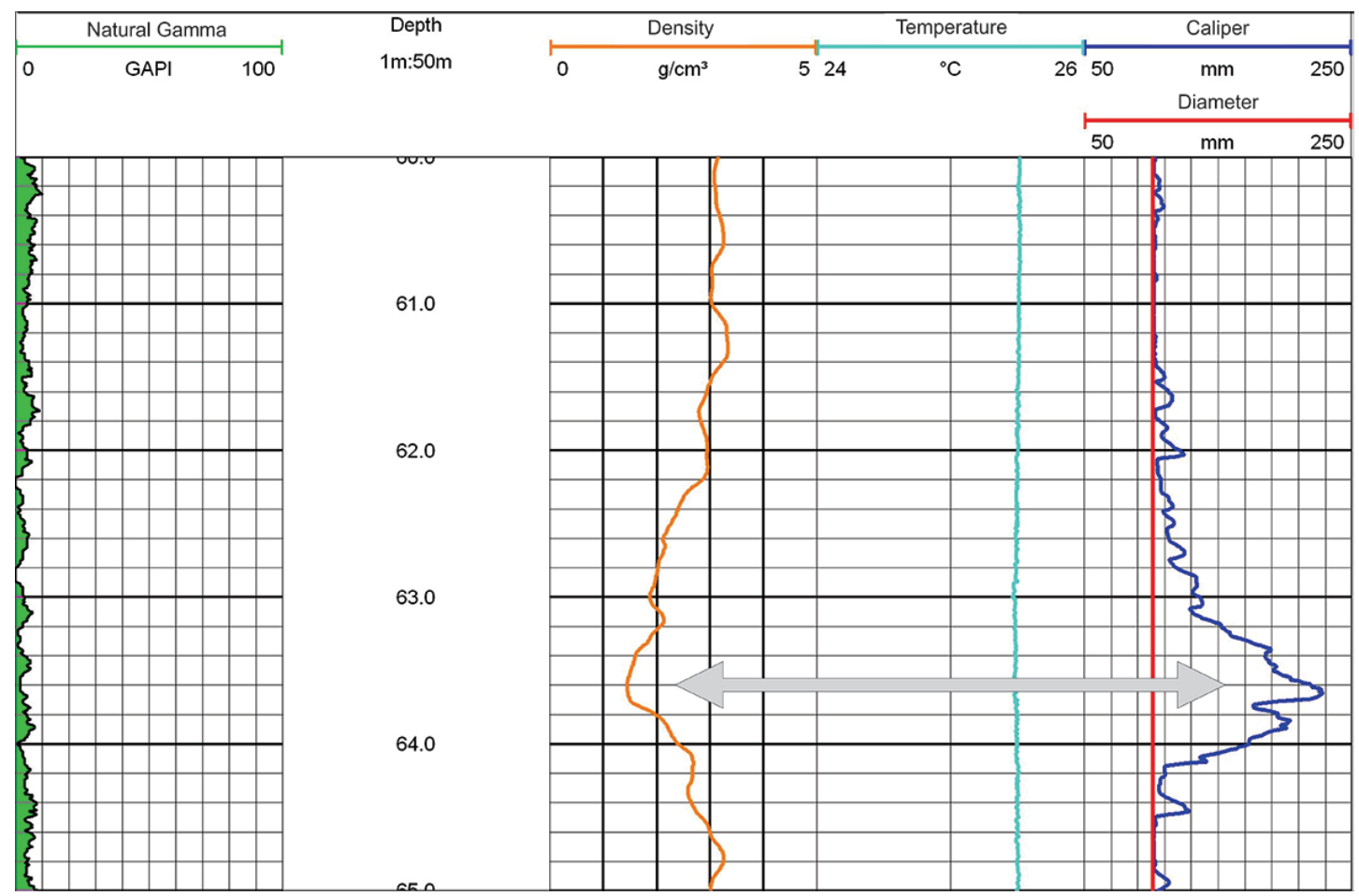

Figure 10 - Example of gamma-gamma profile correlating the borehole wall variations with densities. The arrow in gray indicates the direct correlation between the increase in the diameter of the hole for forcing and consequent decrease in density.

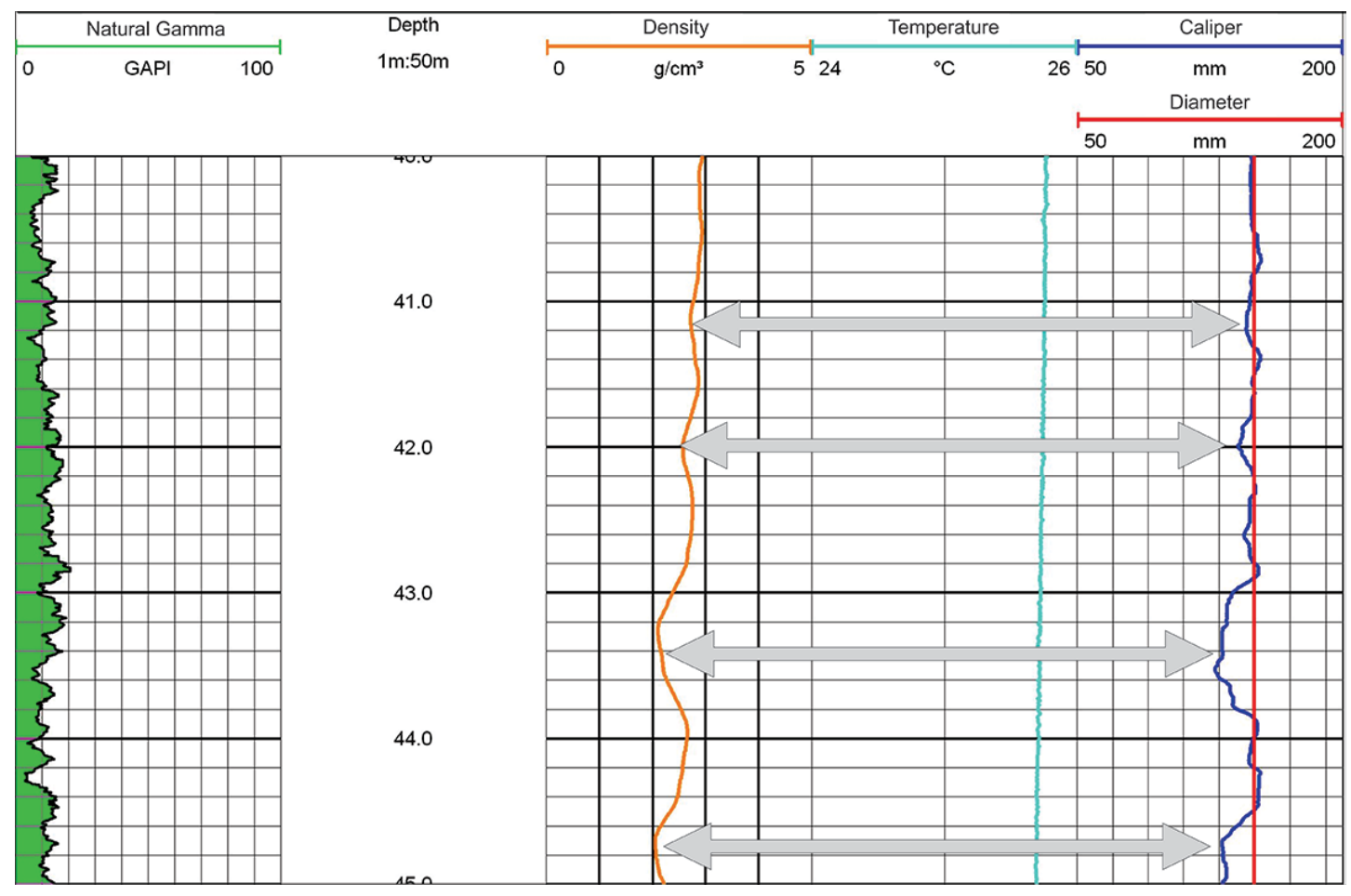

Figure 11 - Example of gamma-gamma profile correlating the borehole wall with densities. The gray arrows indicate the direct correlation between the decrease in borehole diameter and decrease in density. 
the diameter in compact ranges is nearest to the diameter of the drilling tool, and, for the profile of Figure 11, there is no variation in the natural gamma, keeping near $20 \mathrm{API}$, which indicates that there is no variation in the composition of the rock.

Although the logs of Figures 10 and 11 present low counting and certain regularity of natural gamma (no variations above $30 \mathrm{API}$ ), indicative of homogeneous or compact rock, the conditions of the borehole walls cause variations in the density values.

As a way to monitor the validity of a range whose conditions of the borehole walls are sufficiently preserved, and therefore has valid values of density, it is necessary to establish a criterion of tolerance in the diameter of each point measured by the caliper tool.

A validation criterion can be established based on the variation of density associated with the hole walls internal diameter variation, and, for the purposes of quality control, it establishes a maximum diameter variation entailing changes in the value of density within the established error in determining nominal density for each lithology, or alternatively, it may establish the lowest variation between nominal diameter and caliper measurements observed in the dataset.

By means of computational routine, it is possible to define valid ranges based on the observation of caliper data in comparison to the nominal diameter of the hole as shown in Figure 12.

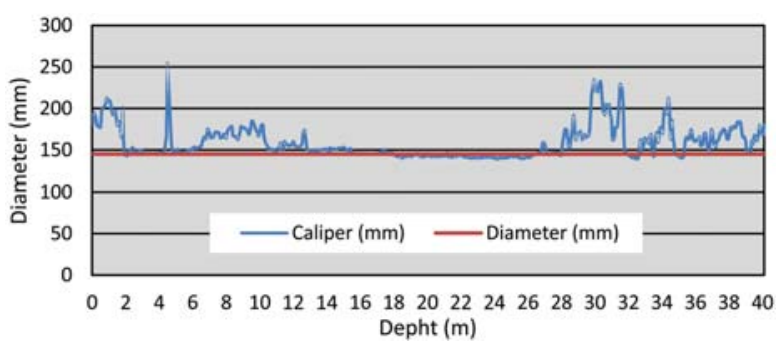

Figure 12 - Comparison between the caliper measurements and a nominal diameter of a borehole.

Due to mechanical factors like the borehole wall roughness, or geological factors, the linearity observed between density measurements of the sensors of short and long spacing, presents spread in the dataset around the adjusted line. Such behavior reflects the non-homogeneous characteristic of the rocks and borehole wall diameters through which sensors of different spacing indicate different densities for the same spot.

This phenomenon is also explained by the fact that, at a same point, the stretch of rock covered by different sensors, occasionally, is different, that is, if the sensors are separated by a distance $x$, at the same time $t$, one of the sensors records the density of the rock in the depth $z$, and the other the density at depth $z+x$.
The difference of the density values with respect to the adjusted line can be decomposed into two components, one in the $x$-axis and other in the $y$-axis denoted by $E_{X}$ and $E_{Y}$. Considering an range of data in which $E_{Y} \ll E_{X}$, that is, the line tends to a horizontal-like behavior, this could still preserve linearity, changing only the slope of the line DSS $\times$ DLS which adjusts the data range.

In this case, by this quality control criteria, such range would be considered valid, even if it is not quality data, as, if $E_{Y} \ll E_{X}$, this means that, at certain stretch of the hole, only one sensor is recording density variations, that is, we will have, for example, DLS measuring the density variations and DSS presenting fixed values over a given range.

In addition, a range with these characteristics may present hidden in the dispersion cloud of dataset around the adjusted line DLS $\times$ DSS.

To identify and separate these ranges from other valid ranges, there is proportionality between the sensors, variations of punctual densities using punctual measure of residuals along the borehole, which is given by the relative differences between DLS and DSS according to Eq. (2).

$$
\varepsilon=\sqrt[2]{D S S^{2}+D L S^{2}}
$$

wherein $D L S$ is the density measurement recorded in the long spacing sensor, and $D S S$ the measurement recorded in the short spacing.

As a criterion of quality control, it is used, for varying difference situations in measurements between the sensors, a tolerance of residuals, based on the error bars associated with the densities of each lithology.

For this, one can consider that the tolerance for residuals is less than or equal to the error associated with only one lithology density measured punctually, or the smallest error in a dataset that contains various lithologies as it is expected for a borehole as shown in Figure 13.

It is possible to see in Figure 13 that, adopting the uncertainty of density values for each lithology (Fig. 13c), the residuals indicate a set of valid ranges, represented by the blue color in Figure 13, limited by this uncertainty, and in the case it takes the lowest uncertainty between a dataset with various lithologies (Fig. 13e), the volume of valid ranges will be less.

Thus, it is expected that, for a set of data without unilateral changes in a continuous range, this presents low values of residuals, indicating no disproportionate variations between sensors.

For the set of data observed in the graph of Figure 8, where one can observe dispersion around the adjusted line DLS $\times$ DSS, the residuals present as shown in Figure 14. 


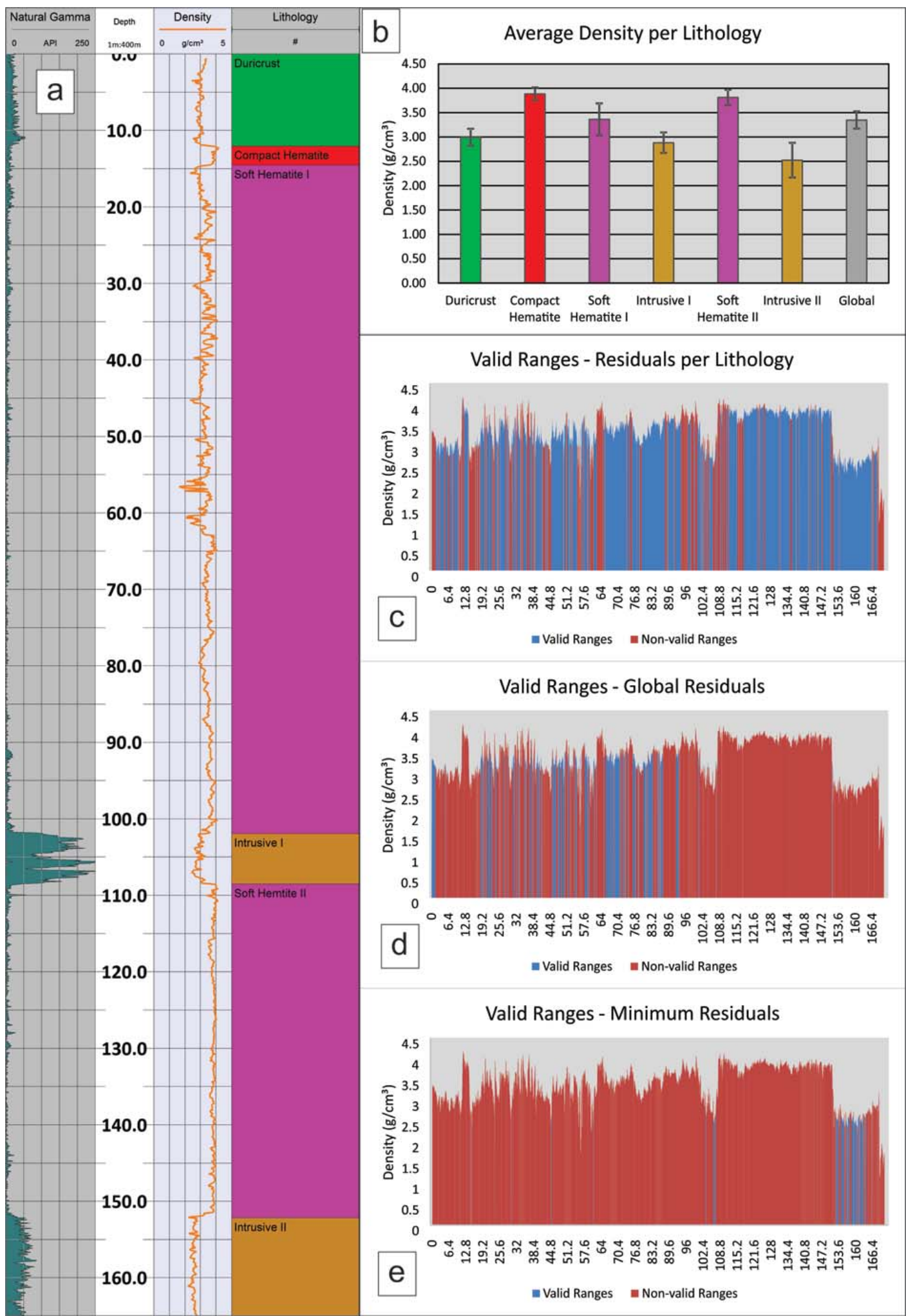

Figure 13 - Example of ranges selection per criterion of residual analysis. (a) Gamma-gamma/ lithological log. (b) Average density per lithology with respective uncertainty. (c) Selection bar considering the uncertainty for each lithology. (d) Selection bar considering a global uncertainty for all lithologies. (e) Selection considering the smaller uncertainty bar among all lithologies. 


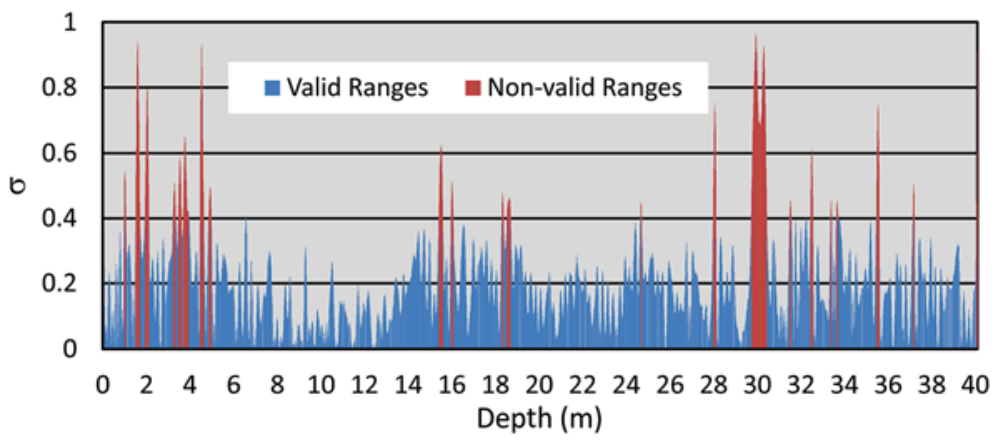

Figure 14 - Residuals recorded for a range of depth in the borehole.

Considering the density values expected for iron formations $\left(3.2 \mathrm{~g} / \mathrm{cm}^{3}\right.$ to $\left.4.1 \mathrm{~g} / \mathrm{cm}^{3}\right)$, the residuals observed in Figure 14 , about $10 \%$ of maximum predicted density, one can establish the criterion of analysis per residuals as a step of quality control, setting a filter in the amount of $0.4 \mathrm{~g} / \mathrm{cm}^{3}$ for the residual over the entire range.

If the residuals are greater than the set value, even if there is linearity, there is no proportionality in the variation of density, that is, a sensor registers more variation in the range than the other.

Thus, control of basic data quality of gamma-gamma well logging applied to the exploitation of ferrous consists of four steps, as follows:

- Verification of linearity between density measurements recorded for the different sensors;

- Verification of the densities range for the entire dataset;

- Comparison between the internal diameter measurements of the borehole compared to the nominal diameter;

- Verification of proportionality in the variation of the density values based on residual analysis.

The four steps should be superimposed, indicating at the end of the analysis, both the valid depth ranges and the non-valid for use in geological mapping.

Once established the parameters applied in the four verification steps, such as limits of the densities range and tolerance in the residual values, the basic steps of quality control may be semi-automated by implementing an integrated computational algorithm.

\section{Processing Flow}

To ensure quality of the gamma-gamma well logging data used for mineral exploitation, it is necessary to proceed, besides the checks proposed as the basic quality control, a verification of the data files of well logging (raw data).
The gamma-gamma well logging data are usually available in .LAS format (Logging ASC// Standard), consisting of a header containing information about the hole, tools used and implementation of roll forming, there may be variations in the content or header format.

Figure 15 shows the data structure with some examples of standard information of a header of .LAS file.

For the implementation of a quality control algorithm for gamma-gamma well logging data, some aspects should be observed with respect to the input data, they are, header formatting, the name of each dataset and the name of dummy data (preset symbolic value assigned to positions where there is no data).

The header should contain all information relating to well logging and the constituent data of the .LAS file, with the example in Figure 15, divided into four items denominated Version Information, Curve Information, Parameter Information and Other Information.

The header item named Version Information, in the example in Figure 15, provides information on the running of geophysical well logging tools that produced the dataset. Among the information in this header item is the name of dummy data, which should be identified and addressed in the quality control algorithm.

The item called Curve Information brings the names of each dataset used in the quality control algorithm to allocate each dataset in a specific function.

The item named Parameter Information contains information about the borehole, which can be used to identify data ranges compromised by the presence of water inside the borehole, which should be considered in the delivery of raw data, or in the algorithm of quality control depending on the number of holes filled with water in a campaign of geophysical well logging.

The item named Other Information presents everything that does not apply to other items, bringing additional information relevant to the processing or interpretation of data. In the example of Figure 15, the item specifies that the well logging was 

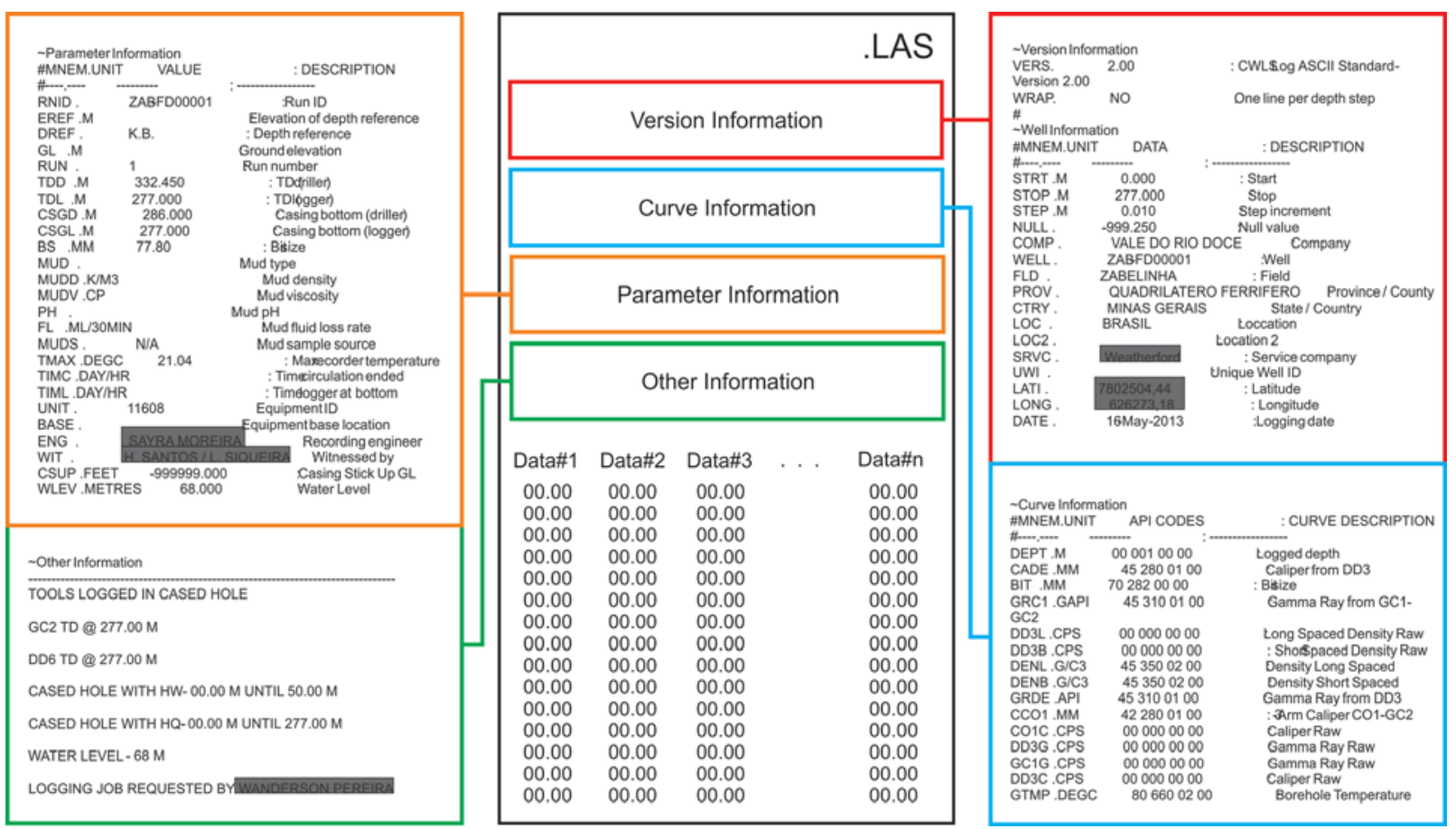

Figure 15 - Structure of a data entry file of geophysical well logging in .LAS format.

performed inside casing (cased hole), which must be considered in quality control so that it proceeds to the specific cased hole data processing.

The formatting of the header should be observed and treated in the algorithm of quality control, considering the possible orders in which the information will be arranged, therefore the mechanism for information tracking.

Another aspect concerning the formatting refers to the separation in the input file, of what will be destined for processing and will be used as metadata in the quality control.

The structure of a control algorithm of gamma-gamma well logging data quality should include three stages, the first being focused on the pre-processing or preparation of the input data to the processing stages, processing the data itself, and consolidation of results.

The pre-processing stage is characterized by adaptation to the input data format and the separation between different datasets for which they are destined to their function in the processing stage.

Data processing has a modular character, in each module is powered by a particular dataset, namely: Linearity check module; Densities range check module; Diameters comparison modules; Residual analysis module.

The final stage, consolidation, compiles the results of each processing module, and presents the results completing the processing flow of the data related to quality control.
The basic structure of a quality control algorithm for data of gamma-gamma well logging is illustrated in the flowchart of Figure 16.

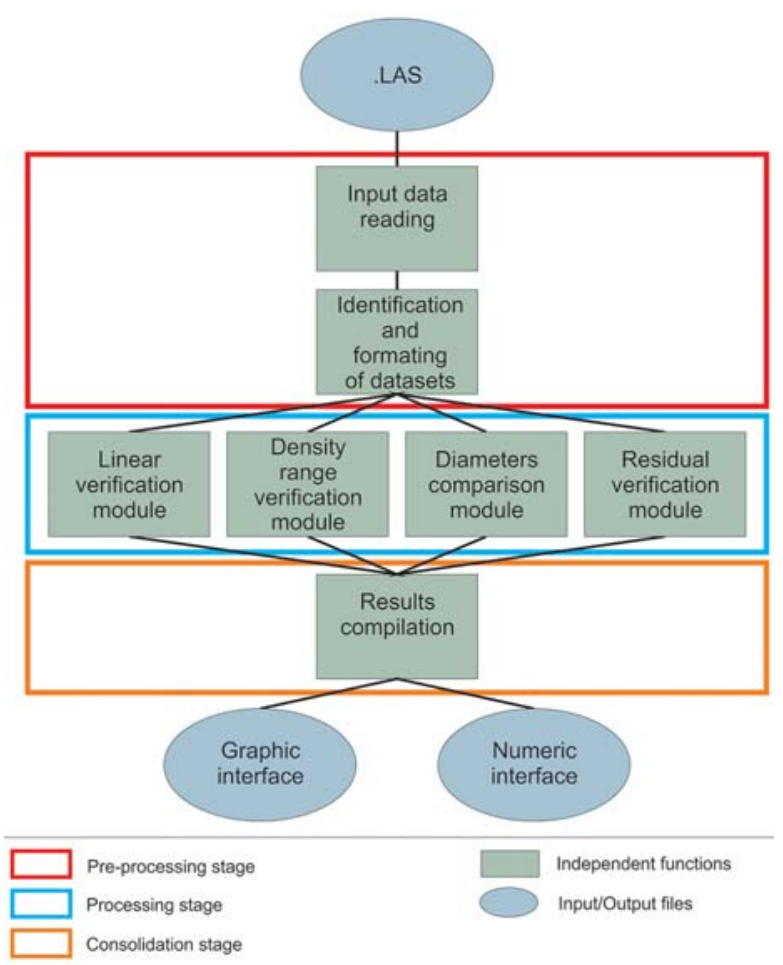

Figure 16 - Flowchart of quality control of gamma-gamma well logging data. 
The implementation of the algorithm illustrated in the flowchart assumes a powerful root program, primarily for reasons of adaptability and adjustments inherent in the development of computational routines, and for the inclusion of additional independent functions as well as the inclusion of the routine as a whole in a more comprehensive external process.

The algorithm can be implemented in stages, which allows specific tests for each function incorporated to the root-program as well as the analysis of the integration of multifunction stages.

Considering the characteristic of multifunction distinct stages, the computational routine should also include the generation and addressing of intermediate input and output files, and the associated computational cost, as a raw data file of gammagamma well logging may contain more than one million data.

\section{RESULTS}

By establishing guidelines based on the above considerations, it is possible to make an efficient computational routine that reads, understands and processes gamma-gamma well logging data focusing on quality control in a semi-automatically manner, making flexible the parameter choices, as the criterion of limit value for residuals, whether it is based on the set of lithologies present in the borehole, or arbitrated by the user.

Another aspect considered in the implementation of the computational routine is the destination of the output files. These are adapted to the intended use of them, as well as the background and needs of the processed data users, taking into account the format in which the data should be arranged and easy of reading for decision making.

When gamma-gamma well logging data is used for geologic modeling, it is interesting that these are presented in numerical form, arranged as spreadsheet data; however, for the purposes of quality control or others requiring wider view of the borehole, a graphic interface as shown in Figure 17 is more appropriate.

Since the functions contained in the processing flow are independent, it is relatively simple to make adjustments to generate hybrids and/or intermediate products.

\section{CONCLUSION}

Considering that:

1- The data observed in the quality control of gammagamma well logging in boreholes if analyzed directly, that is, based only on observation of the roll well logging logs and .LAS files are subject to sensitivity and ability of the data interpreter.
2 - They require considerable computational time, as a borehole with a few hundred meters can produce over one million data.

3 - The quality control process are considered, not one but four distinct aspects in the dataset, linearity between the measurements of different sensors, the range of densities, the comparison of the caliper tool measurements to the nominal diameter, and the proportionality between the measures of the different sensors.

The semi-automation of the quality control allows to restrict any omissions of data interpretation (human factor), and to minimize the computational time.

Therefore, the processing flow facilitate any changes to the criteria, parameters or other factors to be considered in the quality control, and the whole process can be adapted without significant functional implications, both in isolation as in an integrated manner.

The basic quality control proposed in this paper does not exclude, however, a detailed analysis of the data by an interpreter. Such analysis is characterized by well-defined parameters observation, which can be compared with standard values, and, therefore, semi-automated.

The basic quality control consists, therefore a minimal set of observations to attest the quality of gamma-gamma well logging data, after which the data must be analyzed in its entirety, by observing the logs and indications of anomalous features or related to the behavior of the curve, as well as their implications in the interpretation of the data.

\section{ACKNOWLEDGEMENTS}

The authors thank to VALE Iron Ore Exploration for the support and founding of this project.

\section{REFERENCES}

AMIGUN JO \& AKO BD. 2009. Rock Density - A Tool for Mineral Prospection: A Case Study of Ajabanoko Iron Ore Deposit, Okene SW Nigeria. In: The Pacific Journal of Science and Technology, 10(2): 733741.

BALCO G \& STONE JO. 2003. Measuring the density of rock, sand, till, etc. In: UW Cosmogenic Nuclide Laboratory, methods and procedures. Available on: <http://depts.washington.edu/cosmolab/chem.html>. Access on: September 29, 2014.

ELLIS DV. 1987. Nuclear Logging Techniques. In: BRADLEY H (Ed.). Petroleum Production Handbook. 1987. SPE. 

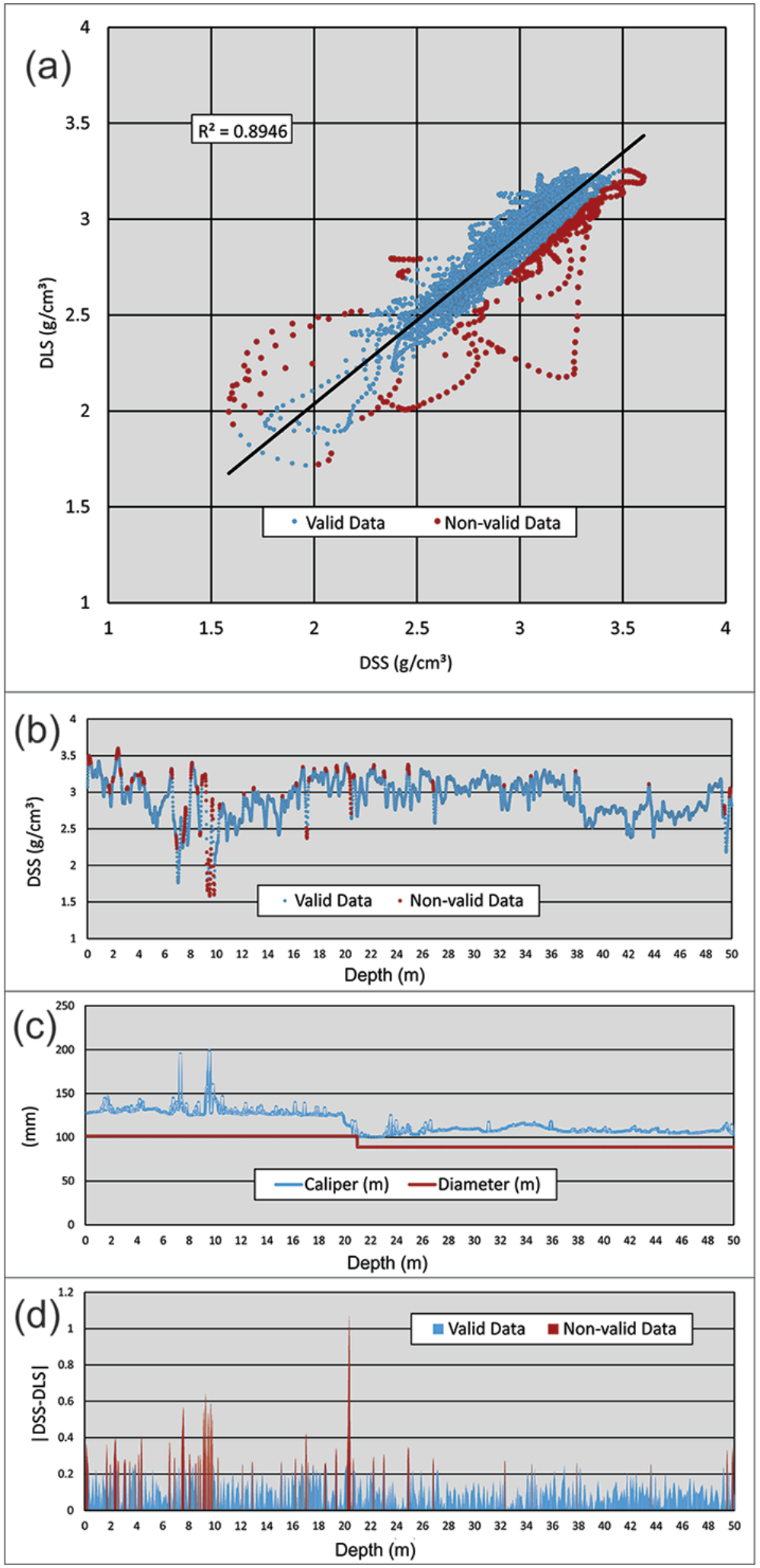

Figure 17 - Example of data output file of gamma-gamma well logging. (a) Verification of linearity between the densities measured by different sensors. (b) Verification of the densities range read. (c) Comparison between the diameters measured by caliper tool and the borehole nominal diameter. (d) Analysis of residuals. 
ELLIS DV \& SINGER JM. 2008. Well Logging for Earth Scientists. Elsevier. $692 \mathrm{pp}$.

KAPLAN I. 1964. Nuclear Physics. Addison-Wesley Publishing Company. 770 pp.

Recebido em 3 novembro, 2014 / Aceito em 6 outubro, 2016

Received on November 3,2014 / Accepted on October 6, 2016
MORGAN KZ \& TURNER JE. 1973. Principles of Radiation Protection. Robert E. Krieger Publishing Company. 662 pp.

TELFORD WM, GELDART LP \& SHERIFF RE. 1990. Applied Geophysics. Cambridge University Press. 792 pp. 